\title{
Knowledge, views, and experience of 25 women with myotonic dystrophy
}

\author{
Claire L Faulkner, Helen M Kingston
}

\begin{abstract}
Twenty-five affected women of reproductive age known to the North West Regional Genetics Family Register (NWRGFR) were interviewed. A semistructured questionnaire, completed by the interviewer, was used to assess understanding and experience of the clinical and genetic aspects of myotonic dystrophy (MD) and attitudes to prenatal diagnosis (PND). Characteristic features of MD (muscle weakness and wasting and myotonia) were well known. Knowledge of other features and complications reflected experience. All subjects were aware that $M D$ is inherited, but only $56 \%(14 / 25)$ knew the risk to their own children and subjects tended to overestimate this risk. Anticipation and maternal transmission of congenital myotonic dystrophy (CMD) were often misunderstood. Almost half of the subjects $(12 / 25)$ perceived themselves to be moderately or severely affected and $40 \%(10 / 25)$ felt that their symptoms restricted daily life. Feelings of devastation, depression, worry about the future, and guilt at the risk of transmission to their children were described. Many subjects (10/25) said that the worst aspect of $M D$ is the risk of transmission to their children. Over half (14/25) said that the risk of transmitting $M D$ had influenced or would influence their own reproduction. Three-quarters of subjects who felt that MD had influenced their reproductive decisions (9/12) chose to limit their family or have no children; only 25\% (3/12) requested PND. Subjects felt that the lack of information concerning clinical severity made PND for MD difficult to consider.
\end{abstract}

(F Med Genet 1998;35:1020-1025)

Keywords: myotonic dystrophy; affected women; reproductive decision making
University

Department of

Medical Genetics and

Regional Genetics

Service, St Mary's

Hospital, Hathersage

Road, Manchester

M13 0JH, UK

C L Faulkner

$\mathrm{H} M$ Kingston Ms Faulkner.

Received 5 January 1998 Revised version accepted for publication 24 April 1998
Correspondence to:

dystrophy arises almost exclusively by maternal transmission, ${ }^{1}$ although rare cases of paternal transmission have been reported. ${ }^{2}$ There is considerable neonatal mortality in congenital myotonic dystrophy (CMD) with moderate to severe physical disability and developmental delay in a high proportion of survivors.

With the identification of the molecular defect responsible for MD in 1992, ${ }^{3-5}$ definitive predictive and prenatal testing became available. While a correlation has been shown between the increase in CTG repeat number and the severity of the disease, the prognosis cannot be determined on the basis of the number of trinucleotide repeats. ${ }^{6}$ The clinical variability in $\mathrm{MD}$ combined with the uncertain genotypic/phenotypic correlation makes genetic counselling very complex, especially with regard to reproductive decisions. These uncertainties can be especially difficult for affected women and their partners because of the particular risk of CMD. It has been suggested that for any female carrying the mutation, the risk of a congenitally affected child is approximately $20 \%$ (of whom half may die in the neonatal period) with $30 \%$ developing myotonic dystrophy at a later stage. However, for a woman with clear multisystem involvement at the time of pregnancy the risk of CMD may be nearer $40 \%$, rising to $50 \%$ once a congenitally affected child has been born. ${ }^{7}$

There have been numerous studies looking at the molecular basis of MD and its associated clinical complications. Few have addressed the experience and opinions of affected subjects. It is known that the presence of a genetic disorder can have significant social and psychological effects on the affected subject and the extended family. ${ }^{8}$ The variable nature of $\mathrm{MD}$ is likely to be particularly difficult for people to cope with, both in terms of their own health and in reproductive decision making. Further insight into affected subject's perceptions of MD and their views about prenatal testing is important for effective genetic counselling.

In view of the particular risk of congenital Myotonic dystrophy (MD) is a progressive, autosomal dominant condition with extreme phenotypic variability. It is the most common muscular dystrophy of adult life with a prevalence estimated at 2.4-5.5 per $100000 .{ }^{1}$ The disease is characterised by myotonia associated with muscle weakness and wasting; however, affected subjects can experience symptoms affecting many body systems. Anticipation occurs in affected families with successive generations tending to present earlier and become more severely affected. There can be a wide range of clinical severity within and between affected families. Congenital myotonic myotonic dystrophy among children of affected women, we interviewed affected women of reproductive age known to the North West Regional Genetic Family Register (NWRGFR). This was a small, descriptive study restricted to a study population of affected women. Many of the issues raised in the study will, nevertheless, be relevant for women "at risk" of inheriting the MD gene. The study sought to assess understanding and experience of the clinical and genetic aspects of $\mathrm{MD}$ among a small group of affected women. Its aim was to gain insight into 
the burden of a variable condition and its influence on reproductive decision making.

\section{Methods}

Established in 1980, the NWRGFR aims to extend genetic counselling from the proband to other at risk and affected family members, to offer annual review by appointment or letter, and to provide continuing counselling and support. MD was added to the register in 1990 and each patient with MD seen in the genetic counselling clinic is offered the services of the register. Placement on the register is voluntary and at the time of ascertainment (April 1994) the register for MD included 103 affected subjects ( 59 females and 44 males) and 55 subjects at $50 \%$ risk of inheriting the MD gene ( 31 females and 24 males). Subjects included on the NWRGFR are asked whether they are willing to be contacted for research purposes at the time of registration.

The study population consisted of the 42 affected women of reproductive age (15-40 years) known to the NWRGFR before April 1994; all had agreed to be approached about participating in research. Subjects were contacted by letter and asked to complete and return a form indicating whether they wished to participate in the study. In view of the relatively small numbers, subjects who had not replied to the initial letter after one month were followed up by telephone to ask if they wished to take part. Subjects who agreed to participate were offered a hospital appointment or home visit. Ethical approval for the study was granted by the Central Manchester Health Authority Clinical Research Ethical Committee.

The study was based on a semistructured questionnaire completed by the interviewer (C Faulkner) at the time of interview. The information gathered was primarily descriptive in nature and the questionnaire was specially designed for the study, drawing on questionnaires used previously to assess understanding and experience in other genetic conditions. ${ }^{89}$ The final study questionnaire contained six sections: (1) background information, (2) knowledge of clinical and (3) genetic aspects, (4) subjects' personal and family experience of $\mathrm{MD}$, (5) reproductive decisions, and (6) attitudes to prenatal testing. A combination of multiple choice and open style questioning was used. Responses to structured questions were coded and percentages calculated for each response. Replies to open questions and additional anecdotes were recorded in writing at the time of interview.

\section{Results}

At the time of ascertainment there were 59 affected women known to the NWRGFR; 42 of these were aged $15-40$ years. Of the 42 subjects identified, 34 were contacted by letter and invited to participate in the study. Three women were not approached because of sensitive family circumstances, two subjects had recently moved out of the North West region, and case notes for three subjects (two kindreds) were unavailable at the time of ascertainment. Of the 34 women contacted by letter, 20 replied and 18 agreed to take part. Fourteen did not reply to the initial contact letter; 10 were contacted by telephone, and seven agreed to an interview. No further contact was possible with four subjects who did not reply to the initial letter. Five women from two kindreds declined to participate (age range 22-34 years). One of these women had an apparently unaffected child and the remaining four were childless. The overall uptake for the study was $74 \%$.

All interviews took place in the subjects' own homes or the home of a relative and lasted one to three hours (mean 1.5 hours). The 25 women interviewed came from 19 kindreds. The age range was $22-40$ years, mean 33 years. The majority of subjects $(21 / 25)$ were married or in long term relationships. Four had divorced; two were in new relationships and two remained single. Eighteen subjects (18/25) had a total of 29 living children (range 1-3). Three subjects from two families had been aware since childhood that MD affected their family and had grown up with some knowledge of the "family illness". For the remaining 22 subjects from 17 families, the family diagnosis was made 3-21 years before interview (mean 9.5 years). The majority of affected women interviewed (13/25) had been investigated on account of known family history; however, four (16\%) presented with symptoms and five $(20 \%)$ were diagnosed after giving birth to a congenitally affected child. Subjects' own diagnoses occurred 3-21 years before interview (mean 9.8 years). On average, subjects were aware of five living affected subjects within their extended family (range 2-12). Information was also gathered on subjects' occupation and religious preference but in this small study there was no obvious relationship between these variables, knowledge of $\mathrm{MD}$, or reproductive decisions and so these data are not presented here.

\section{CLINICAL AND GENETIC KNOWLEDGE}

When asked to name some of the signs and symptoms of $M D$ all subjects $(n=25)$ mentioned muscle weakness, $80 \%(20 / 25)$ mentioned myotonia, and $76 \%(19 / 25)$ said cataracts (table 1). Other signs and symptoms were less well known and tended to reflect subjects' own or family experience. Only a third of subjects (8/25) appeared aware of the risk of anaesthetic complications associated with MD.

Table 1 Symptoms mentioned by the subject group

\begin{tabular}{lll}
\hline Symptom & No & $\%$ \\
\hline Muscle weakness & 25 & 100 \\
Myotonia & 20 & 80 \\
Cataracts & 19 & 76 \\
Speech problems & 10 & 40 \\
Anaesthetic complications & 8 & 32 \\
Digestive problems & 8 & 32 \\
Tiredness & 7 & 28 \\
Aches & 5 & 20 \\
Cardiac involvement & 5 & 20 \\
Hair loss & 4 & 16 \\
Talipes & 4 & 16 \\
Learning difficulties & 4 & 16 \\
Endocrine anomalies & 3 & 12 \\
Auditory problems & 2 & 8 \\
Complications in pregnancy & 2 & 8 \\
Impaired memory & 2 & 8 \\
Other & 2 & 8
\end{tabular}


Table 2 Complications of CMD mentioned by the subject group

\begin{tabular}{lll}
\hline Symptom & No & $\%$ \\
\hline Hypotonia & 14 & 56 \\
Feeding problems & 11 & 44 \\
Respiratory problems & 11 & 44 \\
Motor delay & 10 & 40 \\
Reduced life expectancy & 9 & 36 \\
Talipes & 8 & 32 \\
Learning difficulties & 8 & 32 \\
Speech problems & 5 & 20 \\
Cardiac involvement & 4 & 16 \\
Other & 4 & 16 \\
\hline
\end{tabular}

Subjects were less knowledgeable about the specific complications of CMD (table 2) and subjects with congenitally affected children were more knowledgeable than those without.

Almost all subjects (23/25) knew that symptoms of $\mathrm{MD}$ could vary within affected families and $52 \%(13 / 25)$ knew that the condition is progressive. Five women remarked that children with CMD tend to improve with age; however, none of the congenitally affected children born to this group were over 10 years of age. Other studies ${ }^{1011}$ indicate that the prognosis for infants surviving the neonatal period is good, but declining health in teenage years with death in early adulthood seems likely.

All subjects knew that $\mathrm{MD}$ is an inherited condition, but only $56 \%(14 / 25)$ were able to give the correct risk of transmission to their own children. Of the 11 subjects who answered incorrectly (3/11) or did not know (8/11), none suggested a risk figure below $50 \%$ and all felt the risk was "high". The majority $(17 / 25)$ said that $\mathrm{MD}$ can affect males and females equally although some subjects were often misled by reflecting on the sex and severity of affected subjects within their own families. About half the group (13/25) said that symptoms of $M D$ appeared to worsen in successive generations of a family and $68 \%(17 / 25)$ knew that CMD is more likely when the mother is the affected parent.

Subjects' total knowledge was assessed by awarding points for each correct answer in the clinical and genetic knowledge sections of the questionnaire (range 9-23, mean 16). The maximum score attainable was 32 . Scores were based on points awarded for the main clinical features of $\mathrm{MD}$, and correct answers to questions relating to inheritance of $\mathrm{MD}$, anticipation, and maternal transmission of CMD; higher scores reflected a greater knowledge and awareness of the condition. Total knowledge scores for the group were classified as "high" (range 19-23), "medium" (range 14-18), or "low" (range 9-13). Seven subjects (28\%) obtained a high score, $11 / 25$ subjects (44\%) obtained a medium score, and 7/25 subjects $(28 \%)$ obtained a low score. Subjects with low and medium total knowledge scores had, on average, four affected family members, while those with high scores averaged seven affected relatives. A "severity score" was awarded each subject based on their own perceptions (severely affected $=3$ points, moderately affected $=2$ points, mildly affected $=1$ point). In general, subjects who achieved high total

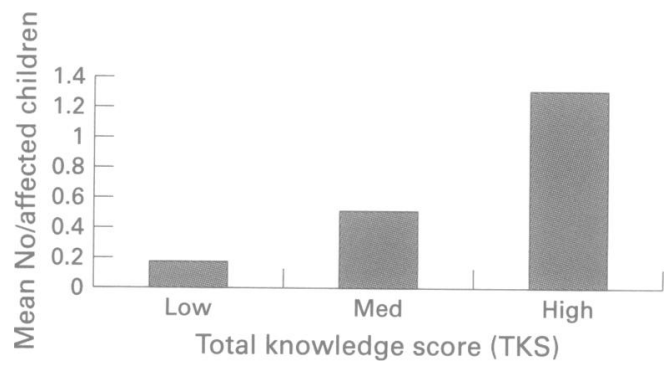

Figure 1 Relationship between the number of affected children each subject has and total knowledge score.

knowledge scores perceived themselves to be more severely affected by $\mathrm{MD}$ (average severity score 1.8) than subjects with medium or low total knowledge scores (average severity score 1.45 and 1.4 respectively). Most striking was the observation that subjects with more clinically affected children achieved notably higher total knowledge scores (fig 1). The possible implications of these observations will be explored more fully in the discussion.

PERSONAL AND FAMILY EXPERIENCE

Subjects were asked how they had felt at the time of their diagnosis. Three women (12\%) said that they were "devastated" and a further seven subjects (28\%) used similar emotive language including "stunned", "worried", "upset", "guilty", and "depressed". Diagnosis was particularly difficult when it occurred after the birth of a congenitally affected child (5/25). One subject said "I could not understand why they were doing so many tests on me when the baby was so sick". Two subjects described feelings of intense guilt that they had been the cause of their baby's problems. Mildly affected subjects who had not given birth to a congenitally affected child appeared to have less difficulty coping with the diagnosis, although some expressed feelings of confusion. Six subjects $(24 \%)$ found it difficult to accept that they had a medical condition of which they had been unaware. One woman said that she had always experienced myotonia of grip but had simply "pulled her fingers straight" and thought no more of it. In common with other studies $^{8}$ subjects often wished to talk at length about the time of diagnosis, with concerns about their own prognosis and feelings of guilt regarding the risk of transmission to children being major themes.

When asked if they felt MD had affected them severely, moderately, or mildly, two women (8\%) said severely, $10(40 \%)$ said moderately, and $13(52 \%)$ said mildly. Ten subjects $(40 \%)$ described restrictions in daily life brought about by physical symptoms of the condition. This included needing help with the housework and shopping, finding it difficult to get out of the house, and suffering from lethargy. Seven subjects (28\%) had particular worries about the future in terms of the health of affected family members, the risk of transmission to children, and the restrictions MD placed on normal family life. Six subjects (24\%) felt that MD had not affected them significantly and two subjects ( $8 \%$ ) said they "just 
Table 3 Worst aspect of $M D$ as reported by the subject

\begin{tabular}{lll}
\hline Worst aspect & No & $\%$ \\
\hline Risk of a child with CMD & 7 & 28 \\
Muscle problems & 5 & 20 \\
Risk of transmission & 3 & 12 \\
Deteriorating health & 3 & 12 \\
Other & 3 & 12 \\
No treatment/cure & 2 & 8 \\
Don't know & 2 & 8 \\
Total & 25 & 100 \\
\hline
\end{tabular}

adapted" and "got on with life". Table 3 shows how subjects responded when asked to name the worst aspect of MD. Other comments included difficulties with speech, loss of memory, and restrictions placed on family life.

REPRODUCTION AND REPRODUCTIVE DECISIONS Eighteen subjects $(72 \%)$ had completed a total of 39 pregnancies (range 1-5) and had a total of 29 living children (range $1-3$ ). Twenty $(51 \%)$ of the 39 pregnancies resulted in offspring who were known to have inherited MD. Sixteen children, born to 10 mothers, were congenitally affected; nine congenitally affected children were still living at the time of interview. Statistically, it could be expected that most of the remaining 19 children (49\%) whose gene status is unknown will not have inherited the $M D$ gene. It is also of interest that $16 / 20$ offspring $(80 \%)$ who were known to have inherited MD were congenitally affected. The relevance of these observations and the possibility of sample bias will be explored in the discussion.

Twelve of the 23 women (52\%) diagnosed before they had completed their family felt that MD had influenced their reproduction. Two women were not diagnosed until they had completed their family and felt that their reproduction might have been influenced. Of the 12 women who had been influenced, all mentioned concerns about the risk of transmission to their children; eight cited the risk of a congenitally affected child in particular and one subject mentioned concerns about her own health in addition to the risk of transmission. Six of those women who had been influenced said that their concerns led them to limit the size of their family, three said that they had decided not to have children, and three had requested prenatal diagnosis.

Nine subjects (39\%) who were diagnosed before completing their family felt that $M D$ had not influenced their reproduction and two (9\%) were still undecided. One subject had requested prenatal diagnosis but maintained that her reproduction had not been influenced by her knowledge of the condition. The child proved to be unaffected and it is possible that the subject responded in this way because she felt able to continue with the pregnancy.

Five of the 11 women who said that their knowledge of $\mathrm{MD}$ had not influenced their reproduction, or who were still undecided, had given birth to congenitally affected children compared to 5/14 women who said that their reproduction had or would have been influenced. Both groups of women achieved similar mean total knowledge scores (16.3 and 16.0 respectively) and 8/11 women who were not influenced or remained undecided felt that they were moderately to severely affected compared to only $4 / 14$ of the group whose reproduction was or would have been influenced. Overall, it appeared that the women whose reproductive decisions were not influenced by their knowledge of MD (or who remained undecided) were well informed about the condition. When questioned further, all reported that their desire for children had outweighed their concerns.

\section{PRENATAL TESTING}

Twenty-one subjects (84\%) were aware that prenatal testing (PND) is available for $\mathrm{MD}$, although only $4 / 25(16 \%)$ had actually requested it. Eighteen subjects $(72 \%)$ felt that the development of PND for MD was a good thing. Four women (16\%) felt that PND might be of benefit to some families but was not for them and three (12\%) were opposed to the offer of PND. Women opposed to PND did not believe in termination of pregnancy and felt that phenotypic variation in MD made termination difficult to consider.

Seventeen subjects $(68 \%)$ felt that they might consider PND themselves in a future pregnancy, but only $9 / 25$ (36\%) felt they would consider termination if the child was shown to have inherited MD. In general, women were interested in "preparing" themselves but felt unhappy about termination in the absence of reliable information about clinical severity. Twenty-three subjects (92\%) felt a prenatal test which could give reliable information about clinical severity would be an improvement and $19(76 \%)$ said that they would consider such a test themselves. All of these women said that they would consider terminating a severely affected pregnancy.

\section{Discussion}

Kessler $^{12}$ suggested that information given within a genetic counselling session is not absorbed in isolation but integrated with previously stored information and experiences. In this study, subjects' knowledge of $M D$ was generally good and related to their own or family experience. Almost all subjects were aware of the main symptoms of $\mathrm{MD}$, its inherited basis, and its variable but progressive nature. Subjects with more affected relatives, those who perceived themselves to be more severely affected, and those with more affected children appeared more knowledgeable about the condition. It seems likely that subjects with more affected relatives/children will have greater first hand experience of MD and will therefore be more knowledgeable. The relationship between knowledge and number of congenitally affected children was particularly striking and may reflect subjects' concern for their offspring as well some possible feelings of guilt about passing the gene on. In view of the intellectual impairment which can be part of the clinical picture in subjects who are more severely affected, it might be expected that subjects who are classified medically as moderately to severely affected could actually be less know- 
ledgeable about the condition, despite having more "first hand experience". It is also possible that subjects with greater knowledge perceive themselves to be more severely affected whereas subjects with less knowledge are less concerned. It would be necessary to compare a medical classification of severity with subjects' own views in order to investigate this further. In their study of patients and families with NF1, Benjamin $e t a l^{\beta}$ found no correlation between subjects' perception of severity and medical classification based on clinical features. A person's perception of the "burden" of genetic disease is likely to reflect psychological areas of concern as well as clinical symptoms of the condition.

Just over half of the subjects $(56 \%, n=14)$ were able to give the correct risk of transmission to their children, somewhat less than in other studies. ${ }^{913}$ Poor or inaccurate recall of information after genetic counselling has been reported by other researchers ${ }^{14}{ }^{15}$ and it is possible that the clinical variability of $\mathrm{MD}$, anticipation, and the maternal transmission of CMD makes it more difficult for subjects to conceptualise the risk of transmission. Anxiety over the risk of CMD may also lead subjects to have a more pessimistic viewpoint and in this study there was a tendency for subjects to overestimate rather than underestimate the risk. A number of the subjects interviewed would have received most of their genetic counselling before the discovery of the genetic mechanism underlying MD. This may account for some of their "lack" of knowledge and may explain much of the confusion surrounding anticipation and maternal transmission of CMD. The detailed information about genetic aspects of MD needs to be presented clearly to aid good understanding by affected subjects; however, recall of genetic "facts" is likely to be complex and not simply related to the clarity of presentation. Summary letters, reinforcement counselling, information leaflets, and continued follow up may be beneficial particularly in facilitating access to genetic counselling services. It is likely that people's circumstances and therefore their ability to integrate aspects of genetic information will change with time. Maintaining contact and the offer of continuing follow up and support is one of the aims of the NWRGFR.

In agreement with other studies on the impact of genetic conditions, ${ }^{8916}$ this study indicates that $\mathrm{MD}$ affects the lives of patients with the condition and their relatives in a variety of ways. The time of diagnosis was particularly difficult for subjects, especially those already coming to terms with feelings of guilt at the birth of a congenitally affected child. Subjects described restrictions in lifestyle brought about by symptoms of their condition and experienced additional worry about the future in terms of their own prognosis and the risk to their children. Variability in the age of onset and clinical presentation of MD adds to the uncertainties for affected subjects and their families.

Ten subjects (40\%) felt that the worst aspect of $\mathrm{MD}$ was the risk to their children and subjects described feelings of guilt at their genetic legacy. Twenty (51\%) of the 39 pregnancies completed by the group were known to have inherited MD. Sixteen $(41 \%)$ of completed pregnancies were congenitally affected, a figure which is similar to the predictions of Koch et $a l^{\prime}$ for women with clear evidence of multisystem disease. Seven of the nine neonatal deaths in this study were the result of complications of CMD. Although it is possible that some of the 19 offspring (49\%) whose genetic status is unknown will go on to develop MD in the future, it would statistically be reasonable to expect that most will not have inherited the MD gene. It is also of interest that $16 / 20(80 \%)$ offspring known to have inherited MD were congenitally affected. It is likely that both these results reflect a degree of subject ascertainment bias, with women at most risk of having congenitally affected children (because of multisystem disease) coming to medical attention and being offered the services of the NWRGFR.

Factors likely to influence reproductive decision making in affected women include knowledge and perception of the risk of transmission, perceived burden of the condition, and the desire for children. ${ }^{17}$ As with other variable adult onset conditions (for example, NF1, APKD), reproductive decision making is complicated by uncertainties surrounding the age of onset and possible clinical severity. The significant risk of a child with CMD makes these uncertainties particularly difficult, a situation which is not helped by the lack of prognostic information obtainable by prenatal testing.

In this study, $12 / 23$ women ( $52 \%$ ) who knew their diagnosis before completing their family said that knowledge of MD had influenced their reproduction. Of those who were influenced, $9 / 12(75 \%)$ responded by limiting their family/deciding not to have children and $3 / 12$ (25\%) requested PND. This indicates that while concerns over the risk of transmission are a real issue, subjects do not necessarily see PND as an acceptable solution. Women whose knowledge of MD had not influenced their reproduction or who remained undecided (11/ 25) were well informed and had similar experience of $M D$ to subjects who had been influenced in terms of numbers of affected family members and numbers of congenitally affected children. There was no correlation between subjects' perception of the severity of the disorder in themselves and influence on reproductive decisions. For women who were not influenced in their reproductive choices, the desire for children outweighed other concerns. Similar findings were reported by Benjamin et $a l^{8}$ in patients with NF1.

Twenty-one subjects $(84 \%)$ were aware that PND is available for $M D$ and $22 / 25$ women $(88 \%)$ felt positive about this availability. Despite this, only $4 / 18$ women $(22 \%)$ who had completed a pregnancy requested PND themselves and only $9 / 25(36 \%)$ felt they might consider PND with termination of an affected pregnancy in the future. Subjects felt that the phenotypic variability in MD made termination of pregnancy difficult to consider; however, 
$19 / 25(76 \%)$ felt that they would consider terminating a severely affected pregnancy. This suggests that women would make different reproductive choices if more information about clinical severity of an affected fetus were available.

This was a small, qualitative study intended to gain insight into the burden of a variable condition and a larger sample group would be needed to allow more complex statistical analysis and estimates of significance. The women included in this study may be more severely affected and have more experience of $M D$ than a group of affected women not ascertained from the NWRGFR. Nevertheless, results from this study give an appreciation of the understanding and concerns of a group of women with myotonic dystrophy and highlight areas of interest both for possible future research and for counselling affected subjects.

1 Harper PS. Myotonic dystrophy. 2nd ed. London: Saunders, 1989.

2 De Die-Smulders CEM, Smeets HJM, Loots W, et al. Paternal transmission of congenital myotonic dystrophy. $\mathcal{F} \mathrm{Med}$ Genet 1997;34:930-3.

3 Harley HG, Brook JD, Rundle SA, et al. Expansion of an unstable DNA region and phenotypic variation in myounstable DNA region and phenotypic

4 Aslanidis C, Jansen G, Amemiya C, et al. Cloning of the essential myotonic dystrophy region and mapping of the putative defect. Nature 1992;355:548-51.
5 Buxton J, Shelbourne P, Davies J, et al. Detection of an untable myotonic dystrophy. Nature 1992;355:547-8.

6 Lavedan C, Hofmann-Radvany H, Shelbourne P, et al. Myotonic dystrophy: size- and sex-dependent dynamics of CTG meiotic instability and somatic mosaicism. Am $f$ Hum Genet 1993;52:875-83.

7 Koch MC, Grimm T, Harley HG, et al. Genetic risks for children of women with myotonic dystrophy. Am 7 Hum Genet 1991;48:1084-91.

8 Benjamin CM, Colley A, Donnai D, Kingston H, Harris R Kerzin-Storrar L. Neurofibromatosis type 1 (NF1): knowledge, experience, and reproductive decisions of affected patients and families. $₹$ Med Genet 1993;30:567-74.

9 Hodgkinson K, Kerzin-Storrar L, Watters A, et al. Adult polycystic kidney disease: knowledge, experience and potitudes to prenatal diagnosis. 7 Med Genet $1990 ; 27 \cdot 552-8$. of childhood onset myotonic dystrophy. Dev Med Child Neurol 1984;26:62-7.

11 Reardon W, Newcombe R, Fenton I, et al. The natural history of congenital myotonic dystrophy: mortality and long term clinical aspects. Arch Dis Child 1993;68:177-81.

12 Kessler S. Genetic counselling: psychological dimensions. New York: Academic Press, 1979.

13 Denayer L, Evers-Kiebooms SG, Van Den Berghe H. A child with CF. I. Parental knowledge about the genetic transmission of $\mathrm{CF}$ and about DNA-diagnostic procetransmission of $\mathrm{CF}$ and about $\mathrm{DN}$

14 Reynolds BD, Puck MH, Robinson A. Genetic counselling: Reynolds BD, Puck MH, Robinson A. G
an appraisal. Clin Genet 1974;5:177-87.

15 Seidenfield MJ, Antley RM. Genetic counselling: a comparison of counsellees' genetic knowledge before and after (part III). Am f Med Genet 1981;10:107-12.

16 DudokdeWit AC, Tibben A, Duivenvoorden HJ, et al. Psychological distress in applicants for predictive DNA testing for autosomal dominant, heritable, late onset disorders. $\mathcal{F}$ Med Genet 1997;34:382-90.

17 Frets PG, Duivenvoorden HJ, Verhage F, et al. Factors influencing the reproductive decision after genetic counselling. Am f Med Genet 1990;35:496-502. 